\title{
Susciter l'engagement des mentors bénévoles pour entrepreneurs : une question de sélection ou d'encadrement ? ${ }^{1}$
}

\author{
Étienne St-Jean, Professeur \\ Institut de Recherche sur les PME, Université du Québec à Trois-Rivières \\ 3351, boul. des Forges, CP 500, 1425 Pavillon Desjardins-Hydro-Québec \\ Trois-Rivières, Qc, Canada G9A 5H7 \\ etienne.st-jean@uqtr.ca \\ Cynthia Mathieu, Professeure \\ Université du Québec à Trois-Rivières \\ 3351, boul. des Forges, CP 500, local 3033, Pavillon Ringuet, \\ Trois-Rivières, Qc, Canada G9A 5H7 \\ Cynthia.mathieu@uqtr.ca
}

Étienne St-Jean détient un doctorat de l'Université Laval et est titulaire de la Chaire de recherche UQTR sur la carrière entrepreneuriale. Il est un membre régulier de l'Institut de recherche sur les PME de l'Université du Québec à Trois-Rivières. Il est aussi éditeur associé de Journal of Small Business and Entrepreneurship. Ses intérêts de recherche concernent le soutien de l'entrepreneur par l'accompagnement et le mentorat, la carrière entrepreneuriale, la personnalité de l'entrepreneur ainsi que les choix stratégiques des dirigeants dans les secteurs forestiers et agricole.

Cynthia Mathieu est professeure agrégée à l'École de Gestion de l'Université du Québec à Trois-Rivières, où elle enseigne le comportement organisationnel et la psychologie industrielle. Ses intérêts de recherche sont liés à la personnalité sombre en milieu de travail (narcissisme et psychopathie en milieu corporatif). Elle a publié des articles dans des revues telles Psychological Assessment et Personality and Individual Differences.

\footnotetext{
${ }^{1}$ St-Jean, É. \& Mathieu, C. (2018) “Susciter l'engagement des mentors bénévoles pour entrepreneurs : une question de sélection ou d'encadrement ?", Revue de l'entrepreneuriat, Vol. 17 No. 3, pp. 139-158. https://doi.org/10.3917/entre.173.0139
} 


\title{
Résumé
}

Les programmes de mentorat pour entrepreneurs reposent sur des bénévoles qui peuvent quitter leur engagement à tout moment. Sachant les coûts reliés à la gestion des programmes et aux formations de ces mentors, l'étude de leur rétention et de leur satisfaction face à leur engagement devient un impératif. Pour étudier cette problématique, nous avons réalisé une enquête auprès de 366 mentors du Réseau $\mathrm{M}$ de la Fondation de l'entrepreneurship (Québec). Nos résultats indiquent que trois des six raisons qui amènent les mentors à s'engager de manière bénévole ont une influence sur leur satisfaction à l'égard de leur engagement ainsi que sur leur rétention : pour comprendre et apprendre, parce que cela correspond à leurs valeurs et pour rehausser leur estime de soi. Il appert que l'investigation des raisons de s'engager pourrait être un outil important dans la sélection des mentors ayant le plus grand potentiel de demeurer actifs et d'être les plus satisfaits dans une organisation de cette nature. Néanmoins, les gestionnaires de programmes de mentorat doivent surtout considérer des activités suscitant la satisfaction envers l'engagement dans la cellule locale pour retenir leurs mentors.

Mots clés : mentorat pour entrepreneur, rétention des mentors, raisons d'être bénévole, satisfaction de l'engagement.

\begin{abstract}
Mentoring programs for entrepreneurs are based on volunteers that can quit their engagement at any time. Knowing the managerial costs of mentoring schemes and those of training mentors, it becomes imperative to study mentor's retention and their satisfaction toward their engagement. To investigate this problematic, we carried out a survey with 366 mentors involved in the Réseau M (M Network) of Fondation de l'entrepreneurship (Quebec). Our results shows that three of six reasons that lead mentors to be engaged as volunteer have an influence on their satisfaction toward their engagement and on their retention: for understanding and learning, because it matches their own values, and for enhancing their self-esteem. It appears that investigating the reasons to engage could be an important tool in selecting mentors with high potential to remain actives and to be the most satisfied within this kind of organization. However, managers of mentoring schemes should mainly consider activities that foster satisfaction toward the engagement in the local mentoring unit to retain their mentors.
\end{abstract}

Keywords: mentoring for entrepreneur, mentor retention, reasons to volunteer, satisfaction with engagement. 


\section{Introduction}

Depuis quelques années, de nombreux programmes d'accompagnement destinés aux entrepreneurs novices ont vu le jour, et ce, dans plusieurs pays du monde. Certains de ces dispositifs, par exemple les programmes de mentorat, font intervenir des bénévoles qui ont acquis une expertise du monde des affaires afin de soutenir les entrepreneurs novices à différents niveaux. Les recherches dans ce domaine indiquent que le mentorat permet à l'entrepreneur novice de développer des apprentissages cognitifs et affectifs, parmi ceuxci la capacité à reconnaître des opportunités et à développer une vision cohérente de son projet d'affaires (Cull, 2006 ; Ozgen et Baron, 2007 ; St-Jean et Audet, 2012 ; Ucbasaran, Westhead et Wright, 2009). Pour que de tels programmes puissent exister et être efficaces, cela implique toutefois d'avoir un bassin de mentors compétents et bien intentionnés pour soutenir les entrepreneurs novices qui réquisitionnent leurs services. En effet, chacun des mentors doit idéalement être formé avant de rencontrer son premier entrepreneur et avoir une rencontre avec le coordonnateur afin de bien comprendre la nature de l'intervention de mentorat, les considérations éthiques de la relation et la confidentialité, et ainsi de suite. Les organisations préfèrent conséquemment conserver leurs mentors, en particulier ceux qui font du bon travail auprès des mentorés, plutôt que de constamment être en mode « recrutement ». L'attrition des mentors est un sujet particulièrement important du fait que certaines relations s'étalent sur plusieurs années et que le mentoré pourrait subir un préjudice en raison du départ prématuré de son mentor, par manque d'intérêt ou autre (Herman et Usita, 1994 ; Madia et Lutz, 2004).

Dans ce contexte, les raisons (ou motivations) qui amènent les mentors à s'engager de manière bénévole dans un programme de mentorat pour entrepreneur pourraient conditionner leur niveau de satisfaction à l'égard de leur engagement ainsi que leur rétention dans le programme. En effet, Okun et al. (1998) proposent six grandes motivations à devenir bénévole dans différents contextes, dont notamment pour fuir ses problèmes et pour faire avancer sa carrière. Ces motivations sont d'ailleurs reliées à des facteurs de succès de la relation dans un contexte d'entrepreneur novice (St-Jean et El Agy, 2012). Il est probable que les mentors s'engageant sur la base de ces dernières motivations pourraient être moins satisfaits de leur participation au programme de mentorat et quitter leur fonction plus rapidement.

La présente recherche visera donc à identifier les facteurs pouvant influencer la rétention des mentors bénévoles pour entrepreneurs, en particulier l'effet des motivations à devenir bénévole sur la rétention subséquente dans le programme de mentorat. Pour y parvenir, nous présenterons brièvement le mentorat pour entrepreneur en tant que pratique, avant d'explorer l'intention de demeurer mentor dans le futur. Par la suite, il sera question des motivations à devenir bénévole, lesquelles pourraient influencer l'intention de demeurer mentor, avant de présenter les compétences utiles ainsi que le rôle de la satisfaction à l'égard de l'engagement dans la cellule. Nous postulons que les motivations à devenir bénévole vont influencer la satisfaction à l'égard de l'engagement dans la cellule de mentorat, et ces deux composantes vont alors influencer l'intention de demeurer mentor dans le futur (effet médiateur de la satisfaction). La méthodologie servant à tester nos hypothèses sera présentée, laquelle fera état de notre recherche auprès de 366 mentors bénévoles du Réseau $\mathrm{M}$, un réseau de mentorat pour entrepreneur situé principalement au Québec (Canada) et des différentes mesures utilisées. Les résultats permettront de constater 
que les motivations à devenir bénévole influencent principalement l'intention de demeurer mentor via un effet médiateur de la satisfaction à l'égard de l'engagement mentoral. Une discussion sera faite de manière à faire ressortir les considérations théoriques et pratiques liées aux résultats obtenus, avant de conclure le présent travail.

1 - Revue de littérature

Cette section présentera le contexte du mentorat pour entrepreneur. Dans un deuxième temps, il sera question de l'intention d'être un mentor bénévole au sein d'un réseau structuré et d'y demeurer, ce qui constitue le point focal de cette recherche. Ensuite, nous présenterons les raisons qui poussent les personnes à devenir bénévole, avant d'aborder les dispositions psychologiques favorables au fait d'être mentor. Pour conclure la section, l'effet important de la structure d'accompagnement sur la rétention du mentor sera exploré, avant de présenter le cadre conceptuel de cette recherche.

\section{1 - Le mentorat pour entrepreneurs}

Depuis quelques années, on assiste à la multiplication des programmes d'accompagnement pour entrepreneur dans le monde, en particulier sous la forme du mentorat. À titre d'exemple, le programme SCORE aux États-Unis, fondé dans les années 1970 et financé par la Small Business Administration (SBA), a soutenu plus de 8 millions de dirigeants de petites entreprises grâce à son réseau de plus de 13000 mentors bénévoles. En Europe, d'autres initiatives similaires existent telles que celle soutenue par le Business Link d'Angleterre, le programme Mentor EgetFöretag de Suède ou France Initiative (en France), avec près de 5000 mentors bénévoles, pour ne nommer que ces programmes-là. Dans presque tous les cas, la formule proposée consiste à jumeler un entrepreneur novice avec un entrepreneur expérimenté et bénévole, ce dernier lui offrant des conseils et des pistes de réflexion visant à lui éviter des erreurs coûteuses, voire fatales.

Tel que le suggère Paul (2004), le mentorat se distinguerait du coaching, du tutorat ou du compagnonnage du fait qu'il est davantage orienté vers la quête de sens plutôt que l'acquisition de techniques. Tout en étant ancré davantage vers l'action, contrairement au counselling, au conseil ou à la médiation, il se distinguerait quand même du parrainage, en étant légèrement moins orienté vers l'action que cette forme d'accompagnement. Ainsi, l'idée sous-jacente au mentorat serait l'éducation, où le mentor est dans un registre de « conduite » et de « guidage », et se distingue du coaching, basé sur l'idée d'entraînement, ou du counselling, axé sur une relation d'aide psychologique (Deschamps, Fatien et Geindre, 2010). Évidemment, il faut souligner que les différentes formes d'accompagnement finissent par se recouper dans la pratique, d'une part, et qu'on en recense néanmoins 13 formes distinctes, d'autre part (D'Abate, Eddy et Tannenbaum, 2003). Pour reconnaître une relation de mentorat, tel que le proposent Haggard et al. (2011), trois dimensions doivent être présentes : il doit y avoir une relation de réciprocité ${ }^{2}$, possédant des bénéfices développementaux pour l'accompagné, en particulier pour la carrière de celui-ci, ainsi que des interactions régulières et substantielles dans une perspective à long terme. De manière plus précise, on estime qu'un mentor pour entrepreneur remplit trois grandes fonctions : les fonctions psychosociales, celles relatives

\footnotetext{
${ }^{2}$ Qui reprend les principes de parité de Paul (2004)
} 
à la carrière de l'entrepreneur novice et la fonction de modèle de rôle (St-Jean, 2010). Soulignons également que le mentor place les intérêts de son mentoré comme une priorité absolue, et non comme faisant partie d'un ensemble de priorités (Gibson, 2005).

\section{2 - L'intention d'être mentor}

L'intention d'être mentor ou de souhaiter le demeurer dans le futur est une problématique relativement nouvelle dans le champ du mentorat mais qui est très importante (Allen, 2007). Elle est liée principalement au fait que les programmes de mentorat sont généralement assez coûteux à mettre en place, malgré le fait qu'ils reposent sur des bénévoles. En effet, dans la grande organisation, du temps doit être consacré à la tenue des rencontres, sans compter les coûts de coordination du programme et les formations des mentors. En contexte entrepreneurial, les coûts sont similaires, à l'exception du temps consacré puisque les mentors ne sont pas rémunérés par l'organisation. Toutefois, les structures d'accompagnement de mentorat ne bénéficient généralement que de peu de ressources qui proviennent en grande partie de l'État, ce qui nécessite une gestion serrée des dépenses. Conséquemment, la rétention des mentors devient particulièrement importante pour ces organisations.

La littérature sur le mentorat en organisation indique que les hommes sont plus enclins à vouloir être mentor, tout comme ceux ayant déjà eu de l'expérience comme mentor par le passé (Ghislieri, Gatti et Quaglino, 2009). On indique également que les traits de personnalité pourraient expliquer l'intention de devenir mentor (Niehoff, 2006). L'expérience de mentorat est également confirmé comme étant une variable importante pour expliquer l'intention de devenir mentor dans d'autres recherches (Allen, Poteet, Russell et Dobbins, 1997; Ragins et Cotton, 1993). Dans le contexte organisationnel, certains mentors souhaitent s'investir pour faciliter leur progression de carrière, étant donné que cette fonction est valorisée et reconnue dans l'organisation, ce qui leur procurera alors de la visibilité (Allen, Poteet, Russell et Dobbins, 1997; Van Emmerik, Baugh et Euwema, 2005). En entrepreneuriat, cette raison n'est forcément pas présente, étant donné que le mentor n'est pas employé dans la structure d'accompagnement. On note également que l'altruisme est positivement relié à l'intention de devenir mentor (Rudnitsky, 2003). Étant donné le peu de récompenses potentielles pour les mentors en contexte entrepreneurial, puisque ceux-ci sont des bénévoles, cette dimension psychologique pourrait être particulièrement importante. À cet égard, les raisons de devenir bénévole pourraient offrir des pistes d'explication quant à l'intention de devenir mentor et, dans le cadre de cette recherche, de vouloir le demeurer dans le futur.

\section{3 - Les raisons de devenir bénévole}

Même si des auteurs indiquent que bien peu de choses sont connues à propos des motivations à devenir bénévole (Bussell et Forbes, 2002; Hunter, 2007), en particulier mentor, quelques travaux sont utiles pour comprendre le phénomène. En effet, des travaux indiquent des sources motivationnelles et dispositionnelles pour expliquer l'intention de s'engager comme mentor bénévole (Allen, 2003; Stukas et Tanti, 2005). Nous abordons ici les sources motivationnelles. À cet égard, nous savons que plusieurs raisons peuvent motiver un individu à s'investir dans une activité bénévole telle que le mentorat pour 
entrepreneur. Selon un rapport de Statistique Canada (2009), les raisons pour lesquelles les personnes font du bénévolat au sein d'un organisme sont les suivantes : le désir de contribuer à la collectivité $(93 \%)$, la volonté de mettre à profit ses compétences et son expérience $(77 \%)$, et le fait d'avoir personnellement été touché par la cause que soutien l'organisme (59\%). D'autres raisons, de moindre importance, concernent découvrir ses points forts $(50 \%)$, constituer un réseau ou rencontrer des gens $(48 \%)$, appuyer l'engagement d'amis (47\%), vouloir améliorer ses perspectives d'emploi (23\%), et s'acquitter de leurs obligations religieuses ou autres croyances (22\%). Robichaud (2003), quant à elle, a relevé les facteurs suivants : se sentir utile, apporter un peu de bonheur aux autres, faire des actions constructives, rendre ce qu'on a reçu, partager avec des amis, nouer des liens et occuper son temps libre.

Cnaan et Goldberg-Glen (1991) postulent que les raisons de faire du bénévolat ne sont pas distinctes, mais qu'elles se croisent. Les personnes bénévoles n'agissent pas pour une seule raison ou une catégorie de raisons, mais par une combinaison de raisons qui peuvent être considérées comme l'ensemble des expériences individuelles enrichissantes (Cnaan et Goldberg-Glen, 1991). Voilà pourquoi il s'avère utile d'utiliser des outils validés de mesure des motivations pour saisir adéquatement les facteurs sous-jacents. De manière plus systématique, Okun et al.(1998) ont testé différentes mesures de motivations à devenir bénévole sur un échantillon de personnes âgées de cinquante ans et plus. Leur objectif était de voir laquelle de ces mesures est la plus prédictive ou explicative des motivations sousjacentes au bénévolat. Selon Okun et al. (1998), le modèle factoriel composé de six (6) facteurs principaux est le plus adéquat, lequel correspond aux raisons suivantes : parce que cela correspond à mes valeurs (l'aide à l'autrui, etc.), par incitation sociale (les relations avec les autres : amis, proches, etc.), pour fuir des problèmes (s'échapper des sentiments négatifs, etc.), pour comprendre et apprendre (s'engager dans des activités qui favorisent l'apprentissage, etc.), pour l'avancement de carrière (la recherche des moyens pour démarrer ou avancer dans la carrière, etc.), et pour rehausser l'estime de soi (obtenir des satisfactions liées à la croissance personnelle et l'estime de soi, etc.). Ces auteurs soulignent que leurs résultats montrent aussi une relation importante entre la motivation par incitation sociale et les personnes plus âgées. Les personnes mariées sont moins motivées à faire du bénévolat pour fuir leurs problèmes par rapport aux personnes célibataires ou divorcées. Les femmes de leur échantillon avaient des scores plus élevés en ce qui concerne la raison « Fuir mes problèmes » par rapport aux hommes.

Dans le contexte du mentorat pour entrepreneur, le fait de s'engager parce que cela correspond aux valeurs du mentor, parce qu'il souhaite comprendre et apprendre et parce qu'il veut rehausser son estime de soi pourrait faire en sorte qu'il souhaite demeurer mentor dans le futur. En effet, plusieurs mentors semblent s'engager pour redonner à une société qui a été généreuse avec eux dans leur vie professionnelle. L'adéquation avec les valeurs pourrait faire en sorte que l'engagement envers le mentorat soit davantage durable. En effet, l'altruisme est une source de motivation forte envers le bénévolat (Burns, Reid, Toncar, Fawcett et Anderson, 2006). Conséquemment, les mentors qui souhaitent redonner aux autres pourraient être davantage satisfaits et vouloir demeurer mentor dans le futur puisque cela touche directement leur motivation. Également, développer une nouvelle relation de mentorat avec un entrepreneur est susceptible d'amener de nouveaux apprentissages pour le mentor, nourrissant l'une des raisons qui pourrait l'amener à s'engager. Le fait d'avoir des tâches qui lui permettent d'apprendre pourraient être une 
source importante de motivation pour le mentor, ce qui est susceptible de le rendre satisfait de son engagement et de le retenir dans son engagement (Janssen, van Vuuren et de Jong, 2014; Millette et Gagné, 2008; van Ginkel, Verloop et Denessen, 2016). Finalement, accompagner un entrepreneur, à plus forte raison si le mentor peut percevoir la progression de celui-ci grâce aux conseils qu'il peut lui prodiguer, est une source importante de valorisation personnelle, nourrissant alors l'estime de soi. Il est aussi démontré que les bénévoles estiment que leur engagement ne fera qu'augmenter leur estime de soi dans le futur (Hibbert, Piacentini et Dajani, 2003), suggérant alors que cela pourrait être une source de rétention dans leur engagement. Ces considérations amènent les hypothèses suivantes :

H1: Les mentors qui s'engagent parce que cela correspond à leurs valeurs auront davantage l'intention de demeurer mentor dans le futur.

H2: Les mentors qui s'engagent pour rehausser leur estime de soi auront davantage l'intention de demeurer mentor dans le futur.

H3: Les mentors qui s'engagent pour comprendre et apprendre auront davantage l'intention de demeurer mentor dans le futur.

Par contre, les raisons pour fuir ses problèmes et pour l'avancement de carrière pourraient ne pas être suffisamment en adéquation avec les impératifs d'une structure d'accompagnement de type mentorat pour donner le goût aux mentors de demeurer actifs dans le futur. D'ailleurs, des travaux montrent que ces raisons ne permettent pas de rendre satisfaits de leur engagement les bénévoles, contrairement à d'autres dimensions (Clary, Snyder, Ridge, Copeland, Stukas, Haugen et Miene, 1998). Aussi, ces deux raisons sont en lien avec des comportements de sortie dans les organisations bénévoles et en particulier, la raison de l'avancement de carrière est reliée négativement avec la rétention (Garner et Garner, 2011). Ces considérations amènent les hypothèses suivantes :

H4: Plus les mentors s'engagent pour fuir ses problèmes, moins ils auront l'intention de demeurer mentors dans le futur.

H5: Plus les mentors s'engagent pour faire avancer leur carrière, moins ils auront l'intention de demeurer mentors dans le futur.

Par ailleurs, parmi les raisons évoquées pour abandonner l'engagement de mentorat, on mentionne les attentes non comblées de part et d'autre, le manque de compétence relationnelle du mentor et le manque de soutien de la part de la structure d'accompagnement (Meissen et Lounsbury, 1981; Spencer, 2007). Cela nous amène vers les compétences essentielles pour être mentor, en particulier la capacité à ouvrir le mentoré ainsi que le contrôle interpersonnel perçu, avant de considérer l'effet de l'implication dans la structure d'accompagnement.

\section{4 - Les profils psychosociaux utiles au mentorat}

De manière générale, les compétences interpersonnelles du mentor sont considérées essentielles au succès d'une relation de mentorat (Clutterbuck, 2004; Pullins, Fine et Warren, 1996) et les mentors qui se considèrent compétents pour être mentor ont de meilleures habiletés interpersonnelles (Bear et Hwang, 2015; Rice et Brown, 1990). Le 
dévoilement de soi, qui réfère à l'habileté de révéler verbalement des informations sur soi (pensées, sentiments et expériences) aux autres (Dindia, 2002) est l'une des nombreuses habiletés qui sont importantes dans le processus de mentorat, autant en contexte organisationnel (Wanberg, Welsh et Kammeyer-Mueller, 2007) qu'entrepreneurial (StJean, 2012). Le dévoilement de soi permet à une relation de s'épanouir seulement lorsque les deux membres de la dyade s'ouvrent, et pas seulement lorsque c'est uniquement le mentoré (Sprecher, Treger, Wondra, Hilaire et Wallpe, 2013). Cela implique donc une certaine ouverture du mentor, certes, mais également une capacité à ouvrir le mentoré. En outre, Wanberg et al. (2007) ont découvert que seul le dévoilement de soi du mentoré (et non celui du mentor) permet à la relation de produire des retombées. D'autres chercheurs ont obtenu des résultats similaires en démontrant que le dévoilement de soi du mentoré permet d'augmenter le mentorat reçu (Blickle, Schneider, Perrewé, Blass et Ferris, 2008). En outre, le dévoilement de soi d'un individu est associé à sa volonté de s'engager dans un programme de mentorat (Pullins, Fine et Warren, 1996). Sachant que le dévoilement de soi du mentoré est important, la capacité à ouvrir les autres serait sans doute une habileté considérée utile pour faciliter le dévoilement de soi des mentorés qui sont davantage discrets (Miller, Berg et Archer, 1983). Un mentor ayant une meilleure capacité à ouvrir les autres pourrait être plus compétent et, du coup, avoir le goût de demeurer mentor dans le futur. Cela fait écho à l'importance des capacités discursives des mentors pour la réussite de cette forme d'accompagnement (Radu Lefebvre et Redien-Collot, 2013; St-Jean et Audet, 2013). Ces considérations amènent l'hypothèse suivante :

H6: La capacité à ouvrir les autres du mentor influence l'intention de demeurer mentor dans le futur.

La théorie du comportement planifié (Ajzen, 1991) postule que les comportements sont guidés par trois sortes de considérations, dont l'une d'elles concerne la croyance à propos des facteurs qui peuvent soutenir ou nuire à la performance du comportement (contrôle comportemental perçu). Selon l'auteur de cette théorie, ces croyances à l'égard du comportement produisent des attitudes favorables (ou non) à l'égard du comportement et mènent à la formation de l'intention comportementale. Selon Ajzen (2002), le contrôle comportemental perçu compte pour beaucoup dans l'explication de la variance de l'intention et de l'action. Il s'agit d'ailleurs d'une des explications importantes de l'engagement bénévole (Wymer, Riecken et Yavas, 1997). Toutefois, les individus peuvent avoir différentes attentes de contrôle selon les différentes sphères de leur vie. Ainsi, Paulhus (1983) propose trois grandes sphères de contrôle liées aux sphères de vie : accomplissements personnels, relations interpersonnelles et influences socio-politiques. Le concept de sphères de contrôle constitue essentiellement une manière plus précise d'aborder la notion de lieu de contrôle. Les attentes de contrôle liées aux interactions avec les autres (notamment en dyade) concernent la sphère interpersonnelle. Ainsi, dans une situation de mentorat, le niveau pour lequel le mentor perçoit qu'il peut contrôler les interactions avec son mentoré réfère à la sphère interpersonnelle de contrôle (Paulhus, 1983). L'internalité du lieu de contrôle (perception qu'un individu peut contrôler les situations) a été associé aux comportements civiques (Blau, 1993). Dans le domaine du mentorat en général, une étude souligne que le lieu de contrôle est associé à la volonté du superviser de devenir mentor (Allen, Poteet, Russell et Dobbins, 1997; Rudnitsky, 2003). Par ailleurs, une méta-analyse démontre que le contrôle perçu par les employés est associé à des hauts niveaux de satisfaction à l'emploi, à l'engagement, à la performance et à la 
motivation, en plus de réduire les intentions de quitter et les départs de l'entreprise (Spector, 1986). Qui plus est, dans le cadre d'une recherche menée auprès de gestionnaires localisés dans 24 entités géopolitiques différentes, il apparaît que la perception de contrôle contribue au sentiment de bien-être de l'individu (Spector, Cooper, Sanchez, O'Driscoll, Sparks, Bernin, Bossing, Dewe, Hart et Lu, 2002). Ces considérations laissent penser qu'un mentor dont la perception de contrôle interpersonnel serait élevée pourrait être davantage compétent pour intervenir auprès de son mentoré ou, à tout le moins, en avoir la perception. En conséquence, cette compétence avérée ou perçue pourrait influencer l'intention de demeurer mentor dans le futur, d'où l'hypothèse suivante :

H7: Le niveau de sphère de contrôle interpersonnelle du mentor influence l'intention de demeurer mentor dans le futur.

\section{5 - L'impact de la structure d'accompagnement sur la rétention des mentors}

Bien que très peu étudié, la structure d'accompagnement pourrait certainement influencer l'intention de demeurer mentor. En effet, il appert que les problèmes liés à la structure influencent négativement les relations de mentorat et l'intention de demeurer mentor dans le contexte du mentorat jeunesse (Spencer, 2007). Tout comme c'est le cas dans les emplois salariés, la satisfaction à l'emploi est largement reconnue comme étant reliée à la rétention des employés (Brigham, De Castro et Shepherd, 2007; Hellman, 1997; Mau, Ellsworth et Hawley, 2008). Le fait de percevoir l'appui de l'organisation envers le mentorat est source d'engagement et de satisfaction de la part des mentors (Hu, Wang, Yang et $\mathrm{Wu}, 2014)$. Par analogie, le fait d'être satisfait de son engagement dans la structure d'accompagnement pourrait certainement conditionner l'intention de demeurer mentor dans le futur. Dans le contexte étudié, plusieurs structures d'accompagnement organisent des rencontres entres les mentors pour échanger sur leurs pratiques, dans une perspective de codéveloppement des savoirs (Payette et Champagne, 1997). Ils vont parfois organiser des rencontres avec les mentorés potentiels, en groupe, afin d'échanger avec eux sur ce qu'est le mentorat et afin de voir s'il y aurait des affinités pour les jumelages subséquents. Ils vont également rencontrer les autres mentors et le coordonnateur à différents moments dans l'année, pour faire le point de l'évolution de la cellule de mentorat ainsi que lorsqu'il y a des formations de dispensées. La satisfaction de l'engagement pourrait agir alors tel un médiateur entre les raisons de devenir bénévoles et l'intention de demeurer mentor. En effet, il est probable que les mentors qui s'engagent pour comprendre et apprendre, parce que cela correspond à leurs valeurs et par incitation sociale pourraient alors être satisfaits de leur engagement dans la cellule de mentorat, cette satisfaction amenant à son tour l'intention de demeurer mentor dans le futur. Cela amène l'hypothèse suivante :

H8: La satisfaction de l'engagement dans la cellule du mentor est un médiateur entre les raisons de devenir bénévole du mentor et son intention de demeurer mentor dans le futur.

La Figure 1 présente le cadre conceptuel de la recherche. Nous pouvons constater que la capacité d'ouvrir du mentor ainsi que son niveau de contrôle interpersonnel devrait influencer son intention de demeurer mentor. Trois des six raisons de devenir bénévole devraient avoir un effet positif sur l'intention, alors que deux devraient avoir un effet négatif. Finalement, la satisfaction d l'engagement dans la cellule agira comme médiateur entre les raisons de devenir bénévole et l'intention de demeurer mentor. 


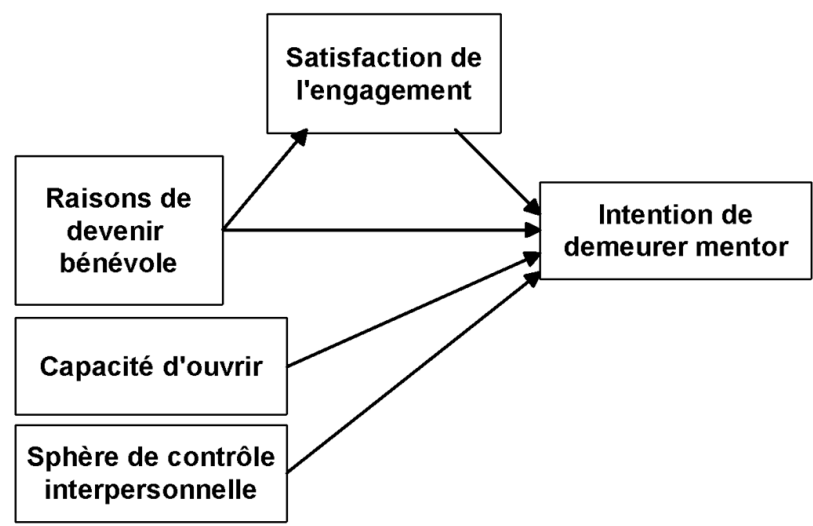

Figure 1. Cadre conceptuel proposé pour la recherche

\section{2 - Méthodologie}

Pour tester les hypothèses proposées, nous avons effectué une recherche auprès d'un programme spécifique, le Réseau M de la Fondation de l'entrepreneurship au Québec, qui sera présenté dans cette section. Ensuite, nous aborderons la population étudiée, soit les mentors de ce réseau de mentorat pour entrepreneurs, ainsi que l'échantillon retenu pour l'analyse. Les variables dépendantes et médiatrices, soit la satisfaction de l'engagement dans la structure ainsi que celles de l'intention de demeurer mentor, seront présentées, avant de poursuivre vers les variables indépendantes, soit les raisons de devenir bénévole, la capacité d'ouvrir ainsi que la sphère de contrôle interpersonnelle (interne). Sachant que certaines variables exogènes peuvent expliquer la rétention, par exemple l'âge du mentor, celles-ci seront justifiées, avant de présenter la stratégie globale d'analyse pour vérifier les hypothèses de recherche.

\section{1 - Le programme étudié}

Le Réseau M (réseau de mentorat d'affaires), qui sert de toile de fond à la présente recherche, a été créé en 2000 par la Fondation de l'entrepreneurship, un organisme voué au développement économique sur le territoire du Québec. Il est offert aux entrepreneurs novices par le biais d'environs 70 cellules de mentorat dispersées sur l'ensemble du territoire du Québec. Ces cellules sont généralement soutenues par des organismes de développement économique tels les Centres Locaux de Développement (CLD), les Sociétés d'Aide au Développement des Collectivités (SADC) et les chambres de commerce locales. Ceux-ci assurent le développement du programme au niveau local ou régional, tout en adhérant au modèle du mentorat d'affaires développé par la Fondation. De façon plus précise, les organismes locaux se dotent d'un coordonnateur de la cellule, lequel est responsable du recrutement des mentors, de l'organisation des séances de formation à leur intention, de la promotion du programme auprès des entrepreneurs novices, du jumelage des participants et de la supervision de la relation qui s'ensuit. Les entrepreneurs novices peuvent bénéficier du soutien d'un mentor pour un coût minime, soit quelques centaines de dollars annuellement, et parfois même gratuitement. Pour le mentor, il s'agit d'une 
démarche totalement bénévole, seuls ses frais de déplacement sont remboursés et, dans bien des cas, ceux-ci ne les réclament pas.

Afin de bien encadrer le développement local, la Fondation de l'entrepreneurship développe des ateliers de perfectionnement orientés sur la relation mentor-mentoré pour éclairer concrètement le rôle des mentors auprès des nouveaux entrepreneurs. Basé sur un code d'éthique d'intervention où la confidentialité des relations est d'une importance capitale, le Réseau M s'est également doté d'un contrat-type pour encadrer et guider les parties dans le choix des modalités de leur relation et des objectifs à atteindre. Il s'agit donc d'un mentorat de type « formel ».

\section{2- La population des mentors et l'échantillon retenu}

Au printemps 2010, le Réseau M de la Fondation de l'entrepreneurship a fourni une liste de 1004 mentors possédant une adresse de courriel valide. À noter que selon les estimations du Réseau M, il y aurait entre 1200 et 1300 mentors. En conséquence, le fait d'utiliser exclusivement une sollicitation par courriel pourrait causer un biais d'échantillonnage puisque les mentors possédant une adresse courriel correspondraient entre $77 \%$ et $83 \%$ de la population totale. Chaque mentor possédant une adresse courriel a reçu une invitation personnalisée à répondre à un questionnaire en ligne ainsi que deux rappels pour les nonrépondants. Ils avaient la possibilité d'obtenir un résumé s'ils complétaient l'enquête. Au total, 366 mentors ont accepté l'invitation, pour un taux de réponse de 36,4\%. Pour une population finie de $1004^{3}$ mentors, l'échantillonnage possède une marge d'erreur de $4,1 \%$, 19 fois sur 20. Nous avons également estimé le biais du non-répondant en comparant les $10 \%$ des répondants les plus hâtifs avec les $10 \%$ des répondants retardataires sur les caractéristiques sociodémographiques et les principales variables du modèle. Suivant l'argument développé par Armstrong et Overton (1977) à l'effet que les retardataires ressemblent davantage aux non-répondants que les hâtifs, et n'ayant constaté aucune différence significative entre ces deux groupes, nous sommes d'avis qu'il n'y a pas de biais du non-répondant dans l'échantillon.

Le Tableau 1 présente les caractéristiques de l'échantillon. On peut remarquer une nette prédominance d'hommes mentors et une scolarité élevée chez ceux-ci, notamment.

\footnotetext{
${ }^{3}$. Rappelons toutefois le biais possible introduit par la technique d'échantillonnage par courriel, excluant les mentors « non branchés ».
} 
Tableau 1. Description de l'échantillon des mentors obtenu

\begin{tabular}{|c|c|c|}
\hline \multirow[t]{2}{*}{ Sexe des mentors } & Homme & $78,5 \%$ \\
\hline & Femme & $21,5 \%$ \\
\hline \multirow[t]{2}{*}{ Âge des mentors } & Moyenne (médiane) & 60,2 ans (61 ans) \\
\hline & Étendue (écart-type) & 33 à 89 ans $(9,22)$ \\
\hline \multirow[t]{4}{*}{ Scolarité } & Secondaire et DEP & $10,9 \%$ \\
\hline & Collégial et technique & $17,1 \%$ \\
\hline & Universitaire $1^{\mathrm{er}}$ cycle & $45,1 \%$ \\
\hline & Universitaire $2^{\text {ème }}$ cycle et + & $26,8 \%$ \\
\hline \multirow{2}{*}{$\begin{array}{l}\mathrm{Nb} \text { d'années d'expérience en } \\
\text { affaires }\end{array}$} & Moyenne (médiane) & 23,56 ans (23 ans) \\
\hline & Étendue (écart-type) & 1 à 54 ans $(11,49)$ \\
\hline \multirow{2}{*}{$\begin{array}{l}\mathrm{Nb} \text { d'années d'expérience en } \\
\text { emploi }\end{array}$} & Moyenne (médiane) & 28,45 ans (31 ans) \\
\hline & Étendue (écart-type) & 1 à 56 ans $(12,02)$ \\
\hline \multirow[t]{2}{*}{ Statut marital } & Célibataire & $18,6 \%$ \\
\hline & En couple & $81,4 \%$ \\
\hline \multirow[t]{2}{*}{ Nombre d'années comme mentor } & Moyenne (médiane) & 4,46 ans ( 3 ans) \\
\hline & Étendue (écart-type) & 1 à 15 ans $(3,30)$ \\
\hline \multirow{2}{*}{$\begin{array}{l}\text { Nombre } \\
\text { (expérience) }\end{array}$} & Moyenne (médiane) & $5,96(4)$ \\
\hline & Étendue (écart-type) & 1 à $21(4,39)$ \\
\hline \multirow{2}{*}{$\begin{array}{l}\text { Recours à un mentor dans le } \\
\text { passé }\end{array}$} & Oui & $41,3 \%$ \\
\hline & Non & $58,7 \%$ \\
\hline
\end{tabular}

2.3 - La variable dépendante et le médiateur

L'examen de la littérature ne nous a pas permis d'identifier de mesures adaptées au contexte concernant ces deux concepts. Nous avons donc proposé des items grâce à une discussion avec les responsables du Réseau $M$ afin de s'assurer que la mesure correspondrait aux réalités étudiées. Pour l'intention de demeurer mentor, nous avons soumis ces trois affirmations aux mentors, qui devaient répondre entre 1-Totalement en désaccord et 7-Totalement en accord : 1-Je souhaite poursuivre mon implication de mentor dans le futur, 2-Les avantages d'être un mentor surpassent les inconvénients, et 3-Je n'hésiterais pas à recommander le mentorat aux autres. Pour la mesure relative à la satisfaction de l'engagement dans la cellule, en utilisant la même échelle que précédemment, le répondant devait se positionner à l'égard de ces quatre affirmations : 1Je suis impliqué dans les activités de la cellule, 2-Je suis satisfait de ma relation avec le coordonnateur, 3-J'apprécie les autres mentors de la cellule, et 4-Je suis satisfait de la fréquence des rencontres avec les autres mentors de ma cellule. 
Afin de vérifier la validité de la mesure, nous avons fait une analyse factorielle des composantes principales avec rotation Varimax en entrant tous les items. Les résultats indiquent la présence de deux facteurs restituant $73,9 \%$ de la variance et les items se classent parfaitement dans les construits théoriques proposés. L'alpha de Cronbach est de 0,855 pour l'intention de demeurer mentor dans le futur tandis que celui de la satisfaction de l'engagement dans la cellule est de 0,837 . Ces résultats suggèrent que les mesures sont suffisamment valides et fidèles pour les analyses subséquentes (Tabachnick et Fidell, 2007).

\section{4 - Les variables indépendantes}

Pour identifier des raisons de devenir bénévole, nous avons utilisé l'outil d'Okun et al. (1998) en 30 items qui propose six (6) raisons différentes pour devenir bénévole (5 items chacune), où le répondant devait indiquer dans quelle mesure l'énoncé répond à sa situation (de 1-Fortement en désaccord à 7-Fortement en accord) : 1-Correspond à mes valeurs, 2-Incitation sociale, 3-Fuir mes problèmes, 4-Apprendre et comprendre, 5Avancement de carrière et 6-Rehausser mon estime de soi. Afin de vérifier la validité de l'outil, nous avons fait une analyse factorielle des composantes principales avec rotation Varimax. Le résultat indique une solution à six facteurs qui explique $70,1 \%$ de la variance totale et dont les items sont classés parfaitement dans les facteurs théoriques proposés par les auteurs. Comme on peut le voir au Tableau 2, les alphas de Cronbach varient de 0,734 à 0,916. Pour les analyses subséquentes, nous avons donc utilisé les scores factoriels, lesquels sont peu corrélés entre eux et orthogonaux. Cette procédure permet de réduire les problèmes potentiels de multicolinéarité dans le cas des régressions linéaires multiples. Toutefois, nous avons également créé des scores moyens pour chacun des facteurs afin de mieux interpréter leur distribution en fonction de l'échelle utilisée (Likert 7). Comme on peut le voir au Tableau 2, la principale raison évoquée par les mentors pour devenir bénévole est pour " comprendre et apprendre », suivi de très près par "correspond à mes valeurs ». Vient ensuite, dans une moindre mesure, pour « rehausser mon estime de soi » et par « incitation sociale », et de manière plus marginale pour « fuir mes problèmes » et pour « l'avancement de carrière ».

Tableau 2 : Information descriptive des raisons de devenir bénévole

\begin{tabular}{lcccc}
\hline \multicolumn{1}{c}{ Facteur (Raison) } & $\begin{array}{c}\text { Moyenn } \\
\mathrm{e}\end{array}$ & Médiane & Écart-type & $\alpha$ Cronbach \\
\hline Comprendre et apprendre & 5,55 & 5,58 & 0,87 & 0,862 \\
Correspond à mes valeurs & 5,50 & 5,60 & 0,76 & 0,734 \\
Rehausser mon estime de soi & 4,65 & 4,80 & 1,14 & 0,862 \\
Incitation sociale & 4,26 & 4,40 & 1,37 & 0,904 \\
Fuir mes problèmes & 2,90 & 2,75 & 1,47 & 0,916 \\
Avancement de carrière & 2,52 & 2,20 & 1,37 & 0,893 \\
\hline
\end{tabular}

Pour la capacité d'ouvrir du mentor, nous avons utilisé l'outil développé par Miller, Berg et Archer (1983) que nous avons traduit. Celui-ci possède 10 items avec des échelles Likert 7. La variable est unidimensionnelle et l'alpha de Cronbach est de 0,914. Pour la sphère de contrôle interpersonnelle, nous avons utilisé la traduction de l'outil développé par Paulhus (1983), qui possède cinq items formulés positivement et cinq autres formulés 
négativement. L'analyse factorielle suggère une structure à deux facteurs, regroupant les items positifs ensembles dans un facteur et les négatifs dans un autre. L'alpha de Cronbach est de 0,73 , ce qui est acceptable.

\section{5 - Variables exogènes}

Certaines variables ont été identifiées comme pouvant potentiellement influencer les résultats (variables exogènes). Ainsi, nous avons retenu le genre, l'âge et la scolarité du mentor, le nombre de jumelages depuis le début de son implication ainsi que sa satisfaction générale reliée à ses relations précédentes (Dans quelle mesure êtes-vous satisfait de vos relations de mentorat?).

\section{6 - Analyse}

Nous avons suivi la procédure proposée par Baron et Kenny (1986) pour tester l'effet médiateur de la satisfaction de l'engagement dans la cellule sur l'intention de demeurer mentor dans le futur. Tel que proposé, nous avons d'abord testé l'effet des variables indépendantes (raisons de devenir bénévole) sur le médiateur (satisfaction de l'engagement), en contrôlant pour les variables exogènes ainsi que la capacité d'ouvrir le mentoré et la sphère de contrôle interpersonnelle (étape 1). Ensuite, nous avons vérifié l'effet des variables indépendantes (raisons de devenir bénévole) sur la variable dépendante (intention de demeurer mentor), toujours en contrôlant pour les variables exogènes ainsi que la capacité d'ouvrir le mentoré et la sphère de contrôle interpersonnelle (étape 2). En dernier lieu, nous avons testé l'effet du médiateur en contrôlant pour celui-ci dans la régression qui comprend l'ensemble des variables d'intérêt (étape 3). Pour qu'un effet médiateur existe, il faut qu'il y ait une relation significative entre les variables indépendantes et le médiateur (étape 1), un effet significatif entre la variable indépendante et la variable dépendante (étape 2) et qu'une fois le médiateur contrôlé (étape 3), l'effet de la variable indépendante sur la variable dépendante soit réduit (effet médiateur partiel), voire annulé (effet médiateur total). Les répondants avec des données manquantes ont été exclus de l'analyse, retenant un minimum de 243 répondants pour la série d'analyse effectuée.

\section{3 - Résultats}

Comme on peut le voir au Tableau 3, les variables de sphère de contrôle interpersonnelle et de capacité d'ouvrir le mentoré ne sont pas significatives pour expliquer la satisfaction de l'engagement dans la cellule. Toutefois, trois des six raisons pour devenir bénévole le sont : pour comprendre et apprendre, pour l'estime de soi et parce que cela correspond aux valeurs du mentor. Le modèle global explique $6,7 \%$ de la variance, ce qui est relativement peu. 
Tableau 3. Régression de la satisfaction de l'engagement dans la cellule

\begin{tabular}{|c|c|c|c|}
\hline & Bêta std. & Bêta std. & Bêta std. \\
\hline Sexe du mentor & 0,061 & 0,069 & 0,034 \\
\hline Âge du mentor & $-0,009$ & $-0,015$ & $-0,003$ \\
\hline Niveau d'éducation & $-0,037$ & $-0,034$ & $-0,023$ \\
\hline Nombre de relations de mentorat & $0,111 \dagger$ & $0,113 \dagger$ & 0,092 \\
\hline $\begin{array}{l}\text { Satisfaction des relations de } \\
\text { mentorat }\end{array}$ & $-0,044$ & $-0,047$ & 0,001 \\
\hline $\begin{array}{l}\text { Sphère de contrôle } \\
\text { interpersonnelle }\end{array}$ & & 0,046 & 0,079 \\
\hline Capacité d'ouvrir le mentoré & & 0,003 & $-0,040$ \\
\hline Comprendre et apprendre & & & $0,207 * *$ \\
\hline Estime de soi & & & $0,195 * *$ \\
\hline Correspond aux valeurs & & & $0,109 \dagger$ \\
\hline Incitation sociale & & & $-0,035$ \\
\hline Fuir ses problèmes & & & $-0,059$ \\
\hline Avancement de carrière & & & $-0,100$ \\
\hline Adj. $R^{2}$ & $-0,005$ & $-0,012$ & 0,067 \\
\hline$\Delta$ Sig. F & 0,584 & 0,794 & 0,000 \\
\hline $\mathrm{N}$ & 243 & 243 & 243 \\
\hline
\end{tabular}

La régression suivante correspond à l'effet des raisons de devenir bénévole sur l'intention de demeurer mentor dans le futur sans effet médiateur (Tableau 4). Comme on peut le constater (modèle 3), plus les mentors sont vieux et moins ils ont l'intention de demeurer mentor dans le futur et tandis que leur expérience de mentorat est reliée positivement et significativement à l'intention de demeurer mentor. La sphère de contrôle interpersonnelle et la capacité à ouvrir le mentoré ne sont toujours pas significatives. Toutefois, trois des six raisons pour devenir bénévole le sont : pour comprendre et apprendre, pour l'estime de soi et parce que cela correspond aux valeurs du mentor. Le modèle global explique $14,1 \%$ de la variance, ce qui est nettement meilleur que pour la précédente régression. Ces résultats confirment les hypothèses $\mathrm{H} 1, \mathrm{H} 2$ et $\mathrm{H} 3$. Par contre, les hypothèses H4, H5, H6 et $\mathrm{H} 7$ ne sont pas confirmées. 
Tableau 4. Régression de l'intention de demeurer mentor dans le futur (sans médiateur)

\begin{tabular}{|c|c|c|c|}
\hline & $\begin{array}{l}\text { Modèle } 1 \\
\text { Bêta std. }\end{array}$ & $\begin{array}{l}\text { Modèle } 2 \\
\text { Bêta std. }\end{array}$ & $\begin{array}{l}\text { Modèle } 3 \\
\text { Bêta std. }\end{array}$ \\
\hline Sexe du mentor & 0,024 & 0,001 & $-0,028$ \\
\hline Âge du mentor & $-0,140 *$ & $-0,124 \dagger$ & $-0,122 \dagger$ \\
\hline Niveau d'éducation & $-0,002$ & $-0,016$ & 0,005 \\
\hline Nombre de relations de mentorat & $0,271 * * *$ & $0,254 * * *$ & $0,221 * * *$ \\
\hline $\begin{array}{l}\text { Satisfaction des relations de } \\
\text { mentorat }\end{array}$ & 0,009 & 0,003 & 0,044 \\
\hline $\begin{array}{l}\text { Sphère de contrôle } \\
\text { interpersonnelle }\end{array}$ & & $-0,039$ & $-0,020$ \\
\hline Capacité d'ouvrir le mentoré & & $0,114 \dagger$ & 0,049 \\
\hline Comprendre et apprendre & & & $0,174 * *$ \\
\hline Estime de soi & & & $0,161 * *$ \\
\hline Correspond aux valeurs & & & $0,197 * * *$ \\
\hline Incitation sociale & & & 0,030 \\
\hline Fuir ses problèmes & & & $-0,036$ \\
\hline Avancement de carrière & & & $-0,057$ \\
\hline Adj. $R^{2}$ & 0,061 & 0,067 & 0,141 \\
\hline$\Delta$ Sig. F & 0,001 & 0,178 & 0,000 \\
\hline $\mathrm{N}$ & 244 & 244 & 244 \\
\hline
\end{tabular}

Finalement, la régression qui inclut le médiateur indique que l'âge du mentor a une influence négative tandis que son expérience de mentorat a un effet positif (Tableau 5). Ni la sphère de contrôle interpersonnelle, ni la capacité à ouvrir le mentoré ne sont significatives dans cette étape ultime (modèle 4). Les hypothèses H6 et $\mathrm{H} 7$ sont définitivement rejetées. On peut voir (au modèle 3) que la satisfaction de l'engagement dans la cellule de mentorat a un effet marqué et positif, ajoutant 0,204 au $R^{2}$ ajusté. Finalement, deux des six raisons pour devenir bénévole sont toujours significatives : pour l'estime de soi et parce que cela correspond aux valeurs du mentor. Cela confirme H1, H2 et $\mathrm{H} 3$ (sur la base des résultats du Tableau 4) et suggère un effet médiateur partiel pour l'estime de soi et parce que cela correspond aux valeurs du mentor, et un médiateur complet en ce qui concerne la raison comprendre et apprendre (Baron et Kenny, 1986). Comme on peut le constater, l'ajout des variables indépendantes (Modèle 3 du Tableau 4) dans la régression sans médiateur ajoute un $R^{2}$ ajusté de 0,074 à l'équation tandis que l'ajout à la régression qui inclut le médiateur (Modèle 4 du Tableau 5) n'est que de 0,022. Qui plus est, la régression sans médiateur possède trois variables indépendantes significatives tandis que celle avec médiateur n'en possède que deux, dont un à $p=0,10$. Ces résultats démontrent la présence d'un médiateur partiel (Baron et Kenny, 1986) et confirme H8. Finalement, l'absence de relation (avec ou sans médiateur) pour les raisons « fuir ses problèmes » et " avancement de carrière » suggère de rejeter définitivement H4 et H5. Le Tableau 6 résume les hypothèses confirmées et rejetées. 
Tableau 5. Régression de l'intention de demeurer mentor dans le futur (avec médiateur)

\begin{tabular}{|c|c|c|c|c|}
\hline & $\begin{array}{l}\text { Modèle } 1 \\
\text { Bêta std. }\end{array}$ & $\begin{array}{l}\text { Modèle } 2 \\
\text { Bêta std. }\end{array}$ & $\begin{array}{l}\text { Modèle } 3 \\
\text { Bêta std. }\end{array}$ & $\begin{array}{l}\text { Modèle } 4 \\
\text { Bêta std. }\end{array}$ \\
\hline Sexe du mentor & 0,032 & 0,009 & $-0,022$ & $-0,028$ \\
\hline Âge du mentor & $-0,146 *$ & $-0,131 *$ & $-0,124 *$ & $-0,129 *$ \\
\hline Niveau d'éducation & 0,002 & $-0,012$ & 0,003 & 0,018 \\
\hline $\begin{array}{l}\mathrm{Nb} \text { de relations de } \\
\text { mentorat }\end{array}$ & $0,279 * * *$ & $0,262 * * *$ & $0,211 * * *$ & $\begin{array}{c}0,198 * * \\
*\end{array}$ \\
\hline $\begin{array}{l}\text { Satisf. des relations de } \\
\text { mentorat }\end{array}$ & 0,010 & 0,003 & 0,025 & 0,044 \\
\hline Sphère de contrôle interp. & & $-0,034$ & $-0,054$ & $-0,045$ \\
\hline $\begin{array}{l}\text { Capacité d'ouvrir le } \\
\text { mentoré }\end{array}$ & & $0,117 \dagger$ & $0,116 *$ & 0,070 \\
\hline Satisfaction de & & & $0,452 * * *$ & $0,402 * *$ \\
\hline l'engagement & & & & $*$ \\
\hline Comprendre et apprendre & & & & 0,084 \\
\hline Estime de soi & & & & $0,092 \dagger$ \\
\hline Correspond aux valeurs & & & & $0,158 * *$ \\
\hline Incitation sociale & & & & 0,055 \\
\hline Fuir ses problèmes & & & & $-0,026$ \\
\hline Avancement de carrière & & & & $-0,016$ \\
\hline Adj. $R^{2}$ & 0,066 & 0,072 & 0,276 & 0,298 \\
\hline$\Delta$ Sig. F & 0,001 & 0,164 & 0,000 & 0,041 \\
\hline $\mathrm{N}$ & 243 & 243 & 243 & 243 \\
\hline
\end{tabular}

$\dagger=p \leq 0,10 ; *=p \leq 0,05, * *=p \leq 0,01 ; * * *=p \leq 0,001$

4 - Discussion

Ayant pris connaissance des résultats dans la section précédente, nous aborderons les contributions théoriques et pratiques de cette recherche et terminerons cette section en faisant ressortir les limites de ce travail.

Tableau 6. Synthèse des hypothèses confirmées ou rejetées

\begin{tabular}{lll}
\hline H1 & $\begin{array}{l}\text { Les mentors qui s'engagent parce que cela correspond à leurs } \\
\text { valeurs auront davantage l'intention de demeurer mentor dans le } \\
\text { futur. }\end{array}$ & Confirmée \\
\hline H2 & $\begin{array}{l}\text { Les mentors qui s'engagent pour rehausser leur estime de soi auront } \\
\text { davantage l'intention de demeurer mentor dans le futur. }\end{array}$ & Confirmée \\
\hline H3 & $\begin{array}{l}\text { Les mentors qui s'engagent pour comprendre et apprendre auront } \\
\text { davantage l'intention de demeurer mentor dans le futur. }\end{array}$ & Confirmée \\
\hline H4 & $\begin{array}{l}\text { Plus les mentors s'engagent pour fuir ses problèmes, moins ils } \\
\text { auront l'intention de demeurer mentors dans le futur. }\end{array}$ & Rejetée \\
\hline
\end{tabular}




\begin{tabular}{lll}
\hline H5 & $\begin{array}{l}\text { Plus les mentors s'engagent pour faire avancer leur carrière, moins } \\
\text { ils auront l'intention de demeurer mentors dans le futur. }\end{array}$ & Rejetée \\
\hline H6 & $\begin{array}{l}\text { La capacité à ouvrir les autres du mentor influence l'intention de } \\
\text { demeurer mentor dans le futur. }\end{array}$ & Rejetée \\
\hline H7 & $\begin{array}{l}\text { Le niveau de sphère de contrôle interpersonnelle du mentor } \\
\text { influence l'intention de demeurer mentor dans le futur. }\end{array}$ & Rejetée \\
\hline H8 & $\begin{array}{l}\text { La satisfaction de l'engagement dans la cellule du mentor est un } \\
\text { médiateur entre les raisons de devenir bénévole du mentor et son } \\
\text { intention de demeurer mentor dans le futur. }\end{array}$ & Confirmée \\
\hline
\end{tabular}

\subsection{Contributions théoriques de la recherche}

Les résultats obtenus sont intéressants à plusieurs égards. D'abord, il est maintenant démontré que les raisons de devenir bénévole ont un effet sur la satisfaction de l'engagement du mentor et sa rétention dans le contexte du mentorat pour entrepreneur. Ainsi, le fait qu'une personne s'engage comme mentor pour entrepreneur parce que cela lui permet de comprendre et d'apprendre, parce que cela correspond à ses valeurs ou pour rehausser son estime de soi permet de prédire sa satisfaction à l'égard de son engagement dans la cellule de mentorat et son intention de demeurer mentor dans le futur. Sachant que ces raisons ont également une influence pour expliquer la confiance que portera le mentoré à son mentor (St-Jean et El Agy, 2012), ce concept devient alors d'un intérêt certain pour la littérature sur le mentorat dans différents contextes où des bénévoles sont sollicités, par exemple le mentorat jeunesse (Herman et Usita, 1994; Madia et Lutz, 2004; Meissen et Lounsbury, 1981; Morrow et Styles, 1995).

Ensuite, le fait de vouloir être bénévole pour fuir ses problèmes ou pour l'avancement de la carrière n'a pas d'effet négatif sur la satisfaction de l'engagement et la rétention des mentors, malgré ce qui avait été anticipé. Il est possible que le peu de variance dans les résultats soit en cause, ces deux variables avaient des scores moyens beaucoup plus bas que les autres raisons. Il est également possible que certains mentors qui souhaitaient fuir leurs problèmes puissent avoir trouvé une occupation intéressante dans la cellule de mentorat. Toutefois, ceux-ci ne sont sans doute pas assez nombreux pour que cela ait pu influencer globalement la variable relative à la rétention ou à la satisfaction puisque les résultats ne sont pas significatifs. La même chose pourrait se produire auprès des mentors s'investissant par avancement de carrière. Sachant que certains mentors sont également consultants ou coach et, du coup, rémunérés lorsqu'ils interviennent auprès d'entrepreneurs, il est possible que certains se servent de leur position de mentor pour recruter des clients, bien que le code d'éthique stipule qu'il n'est pas possible de procéder de la sorte et que les coordonnateurs sont vigilants à cet égard. Si de tels mentors existaient, il est possible qu'ils puissent être satisfaits de leur engagement et qu'ils aient l'intention de demeurer mentor puisqu'ils en tireraient des bénéfices. De tels mentors pourraient venir «brouiller » la relation linéaire entre les variables et empêcher de voir une relation significative.

Les résultats obtenus apportent un éclairage intéressant sur d'autres dimensions relatives à la satisfaction de l'engagement ainsi qu'à la rétention des mentors. D'abord, aucune autre variable utilisée dans cette recherche autre que les raisons de devenir bénévole 
se sont avérées significatives pour expliquer la satisfaction de l'engagement dans la structure. Toutefois, en ce qui concerne la rétention des mentors, nous avons pu constater que l'âge possède un effet négatif. Cela indique que plus les mentors sont vieux, moins ils ont l'intention de demeurer mentor. Évidemment, avec un âge médian de 61 ans, il est possible qu'à partir d'un certain âge, la rétention devienne de plus en plus difficile. Également, le nombre de relations de mentorat est fortement relié à la rétention. Il est fort possible que le fait d'avoir eu plusieurs relations par le passé, combiné au fait que le mentor soit toujours actif (puisqu'il répond au questionnaire), laisse présager que cette variable pourrait être, à certains égards, un indicateur de rétention. En effet, si le mentor continue d'être dans la structure après avoir eu un certain nombre de jumelage, c'est qu'il apprécie son engagement de mentor et risque d'avoir le goût de poursuivre dans le futur. Cependant, la satisfaction des relations de mentorat n'est par reliée à l'intention de demeurer mentor dans le futur. Ce résultat est surprenant puisqu'il était attendu que cette variable puisse avoir un effet. Il est possible que la variable relative au nombre de relations de mentorat soit en cause dans cette observation, celle-ci allant chercher une partie de la variance de l'intention de demeurer mentor dans le futur. Cela pourrait réduire la taille de l'effet observé à propos de la satisfaction à l'égard du mentorat et, du coup, d'obtenir un résultat statistiquement significatif.

Au niveau de l'effet médiateur, les résultats sont instructifs d'un point de vue théorique et pratique. En effet, nos résultats démontrent que sur les trois raisons significatives pour expliquer la rétention des mentors (i.e. comprendre et apprendre, parce que cela correspond à mes valeurs, et pour l'estime de soi), seule pour comprendre et apprendre a un effet médiateur complet. Cela veut dire que cette raison n'a pas d'effet direct sur la rétention, mais elle agit plutôt sur la satisfaction de l'engagement mentoral, cette dernière ayant un effet sur la rétention. L'effet médiateur de la variable estime de soi est aussi très fort, cette variable n'ayant un effet direct significatif qu'à un seuil de $p=0,10$. Ainsi, cela veut dire que pour étudier la rétention des mentors, il est essentiel de considérer la satisfaction de l'engagement, et de considérer les variables liées aux raisons de devenir bénévole comme étant davantage en amont de la rétention. D'un point de vue pratique, cela veut aussi dire que les organisations qui mobilisent des mentors bénévoles ne doivent pas se limiter à explorer les raisons qui amènent ceux-ci à s'engager dans de telles relations, mais plutôt travailler à améliorer la satisfaction à l'engagement dans la cellule de mentorat. À cet égard, les activités de formation pour les mentors, tout comme les ateliers de codéveloppement ou même les activités sociales sont autant de pistes à explorer pour comprendre comment se bâtit la satisfaction envers la cellule de mentorat, qui est la clé principale à la rétention. Dans d'autres contextes, ces activités de développement professionnel des mentors s'avèrent importantes (Langdon, 2014).

De manière surprenante, la capacité d'ouvrir le mentoré ainsi que le contrôle interpersonnel, deux qualités pouvant être importantes pour le mentor, ne sont pas significatives pour expliquer la rétention de ceux-ci. Il est probable ici que les liens entre ces qualités et la rétention soient plus complexes et indirects. En effet, pour que ces qualités puissent être utiles, il faut que le mentor puisse avoir eu des mentorés qui étaient « difficiles à ouvrir », ce qui n'est peut-être pas le cas de manière suffisamment fréquente dans le contexte étudié. Il est possible que ces qualités aient des impacts sur les retombées de la relation de mentorat, en développant la confiance ou la similitude perçue (St-Jean, 2012), ce qui n'a pas été mesuré ici. Il faut souligner que la capacité d'ouvrir le mentoré était 
significative dans les modèles incomplets qui ne tenaient pas compte des raisons de devenir bénévole. Cela veut dire qu'en comparaison aux raisons de devenir bénévole, cette variable n'est que marginale pour expliquer la satisfaction de l'engagement ou la rétention.

Finalement, la satisfaction à l'égard de l'engagement dans la cellule est une variable médiatrice partielle de la relation entre les raisons de devenir bénévole et la rétention des mentors. Ce résultat est intéressant puisqu'il permet de mettre en lumière le fait que la satisfaction à l'égard de l'engagement dans la cellule est conditionnée par les mêmes facteurs que la rétention des mentors, à savoir trois des six raisons de devenir bénévole. Ce résultat trouve écho dans d'autres contextes que l'entrepreneuriat, où les raisons liées à l'estime de soi et la concordance avec les valeurs sont reliées à la satisfaction de l'engagement (Dwyer, Bono, Snyder, Nov et Berson, 2013). Là aussi, ce résultat est susceptible d'alimenter les travaux réalisés auprès de bénévoles mentors dans d'autres contextes que l'entrepreneuriat. Il faut aussi rappeler que le peu de variance expliquée par le modèle suggère que d'autres facteurs, non considérés dans cette recherche, pourraient mieux expliquer la satisfaction de l'engagement et, conséquemment, la rétention. À ce sujet, on peut penser que la gestion des bénévoles au sein de la structure par le coordinateur (Hu, Wang, Yang et $\mathrm{Wu}, 2014)$ et les activités mises en place pour soutenir le développement des compétences du mentor (Bakkali, Messeghem et Sammut, 2010) sont des pistes intéressantes à investiguer dans le futur.

\subsection{Contributions pratiques de la recherche}

Le fait de connaitre que trois raisons de devenir bénévole sont en relation avec la satisfaction de l'engagement dans la structure d'accompagnement et rétention du mentor suggère l'utilisation de l'outil d'Okun et al. (1998) lors de la sélection des mentors. En effet, les structures d'accompagnement gagneraient à sélectionner les mentors qui ont le meilleur potentiel de satisfaction à l'égard de leur engagement dans la cellule ainsi qu'au niveau de l'intention de demeurer mentor dans le futur. Ainsi, les efforts de recrutement et les coûts de formation et de gestion du programme seraient optimisés.

De plus, la rétention des mentors est fortement influencée par la satisfaction de l'engagement dans la structure. Cela indique aux organisations qui gèrent des programmes de mentorat pour bénévoles de mettre en place des activités qui rendent les mentors satisfaits de leur engagement. Au sein du Réseau M de la Fondation de l'entrepreneurship, chaque cellule développe des activités qui lui sont propres, que ce soit des déjeuners entre mentors et mentorés, la présence de mentors auprès de différentes organisations pour promouvoir le mentorat, la participation à des comités nationaux de mentors régionaux, etc. De plus, plusieurs cellules ont mis en place des activités de codéveloppement entre pairs, où le chef-mentor anime les échanges à propos de situations vécues lors des accompagnements. Ainsi, un partage d'expériences s'opère et permet aux mentors d'améliorer leurs pratiques. Cela rejoint les travaux menés dans les structures d'accompagnement qui mettent en évidence le besoin de développer les compétences des personnes accompagnées, notamment en ce qui concerne le savoir-être (Bakkali, Messeghem et Sammut, 2010). Ces activités, en plus d'être utiles au développement des compétences mentorales, permettent sans doute aux mentors de se sentir engagés dans la structure, renforçant leur satisfaction à cet égard.

\subsection{Limites de la recherche}


Évidemment, cette recherche n'est pas exempte de limites. Premièrement, les mesures de rétention et de satisfaction de l'engagement dans la structure ont été développées pour cette recherche. Même si les items possèdent une certaine validité apparente, non seulement d'un point de vue logique dans leur formulation même qu'au niveau des tests effectués, ces mesures ne sont sans doute pas exemptes de biais potentiels. Une mesure plus objectivable de rétention aurait pu permettre d'en améliorer la validité. Deuxièmement, il est possible que les deux dimensions psychologiques du mentor, à savoir la capacité d'ouvrir ainsi que l'internalité de la sphère de contrôle interpersonnel, puissent avoir un effet indirect sur la rétention du mentor au travers l'auto-efficacité relative à la posture mentorale, un élément qui n'a pas été considéré dans cette recherche. Finalement, la satisfaction à l'égard de la structure pourrait s'expliquer par de nombreux facteurs autres que les raisons de devenir bénévole, par exemple les activités de codéveloppement et sociales organisées par la cellule. N'ayant pas sous la main les variables appropriées pour bonifier les analyses, l'absence de celles-ci pourrait expliquer la faible variance expliquée. Une analyse plus complète à cet égard serait souhaitable pour le futur.

Conclusion

Cette recherche a permis de voir l'importance de comprendre les raisons qui amènent les mentors pour entrepreneurs à s'engager comme bénévoles. Ces éléments influencent à la fois la satisfaction à l'égard de la structure d'accompagnement ainsi que la rétention des mentors. Pour les recherches futures, sachant que la formation des mentors est fondamentale pour le développement de retombées du mentoré (St-Jean et MitranoMéda, Accepté), il serait intéressant de vérifier si les raisons de devenir mentor influencent le nombre de formation suivies. Ces raisons pourraient avoir une incidence sur de nombreux autres éléments de la relation, que ce soit le style du mentor ou même les fonctions exercées par celui-ci (St-Jean et Audet, 2013; Wanberg, Welsh et Hezlett, 2003). Le fait que la satisfaction à l'égard de la structure soit fortement reliée à la rétention suggère de mieux comprendre ce qui pourrait rendre les mentors satisfaits à cet égard, au-delà des raisons de vouloir être bénévole, notamment au niveau des activités pouvant être mises en place par la structure. En outre, sachant que les comportements prosociaux des mentors expliquent la rétention de ceux-ci dans leur engagement (Bear et Hwang, 2015), d'une part, et que certaines dispositions psychologiques sont utiles pour comprendre le succès d'une relation de mentorat, d'autre part, l'investigation d'autres dimensions psychologiques et liées à la personnalité sont des voies de recherche pertinentes. À cet égard, on peut penser à l'orientation dans un but d'apprentissage (Egan, 2005; Kim, 2007; St-Jean et Tremblay, 2011), au style d'attachement (Wang, Noe, Wang et Greenberger, 2009), au style cognitif (Armstrong, Allinson et Hayes, 2002), au narcissisme ou à la modestie (Allen, Johnson, $\mathrm{Xu}$, Biga, Rodopman et Ottinot, 2009; Blickle, Schneider, Perrewé, Blass et Ferris, 2008) ou à la personnalité (Niehoff, 2006) comme des dimensions qui pourraient être éclairantes, par exemple. Il s'agit de pistes potentiellement prometteuses et qui pourraient confirmer davantage l'utilité de la sélection des mentors pour les organisations gérant les programmes de mentorat ainsi que la pertinence des activités déployés à leur égard. 


\section{Références}

AJZEN, I. (1991), «The theory of planned behavior », Organizational Behavior and Human Decision Processes, vol. 50, $\mathrm{n}^{\mathrm{o}}$ 2, p. 179-211.

AJZEN, I. (2002), « Perceived Behavioral Control, Self-Efficacy, Locus of Control, and the Theory of Planned Behavior », Journal of Applied Social Psychology, vol. 32, $n^{\circ} 4$, p. 665-683.

ALLEN, T. D. (2003), « Mentoring others: A dispositional and motivational approach », Journal of Vocational Behavior, vol. 62, $\mathrm{n}^{\mathrm{o}}$ 1, p. 134-154.

ALLEN, T. D. (2007), "Mentoring Relationships From the Perspective of the Mentor », dans K. E. Kram et B. R. Ragins (dir.), The Handbook of Mentoring at Work: Theory, Research, and Practice, Thousand Oaks, CA, Sage Publication, p. 123147.

ALLEN, T. D., JOHNSON, H.-A., XU, X., BIGA, A., RODOPMAN, O. B. et OTTINOT, R. C. (2009), " Mentoring and protégé narcissistic entitlement », Journal of Career Development, vol. 35, nº 4, p. 385-405.

ALlEN, T. D., POTEET, M. L., RUSSELL, J. E. A. et DOBBINS, G. H. (1997), « A Field Study of Factors Related to Supervisors' Willingness to Mentor Others », Journal of Vocational Behavior, vol. 50, $\mathrm{n}^{\circ}$ 1, p. 1-22.

ARMSTRONG, J. S. et OVERTON, T. S. (1977), " Estimating nonresponse bias in mail surveys », Journal of Marketing Research, vol. 14, no 3, p. 396-402.

ARMSTRONG, S. J., ALLINSON, C. W. et HAYES, J. (2002), «Formal Mentoring Systems: An Examination of the Effects of Mentor/Protégé Cognitive Styles on the Mentoring Process ", Journal of Management Studies, vol. 39, nº 8, p. 11111137.

BAKKALI, C., MESSEGHEM, K. et SAMMUT, S. (2010), « Les structures d'accompagnement à la création d'entreprise à l'heure de la gestion des compétences », Management \& Avenir, n 39, p. 149-162.

BARON, R. M. et KENNY, D. A. (1986), « The Moderator-Mediator Variable Distinction in Social Psychological Research: Conceptual, Strategic, and Statistical Considerations ",, Journal of Personality and Social Psychology, vol. 51, no 6, p. 1173-1182.

BEAR, S. E. et HWANG, A. (2015), « Who mentors? Contextual prosocial motivation and willingness to be a mentor », Human Resource Development International, vol. $18, n^{\circ} 1$, p. $58-75$.

BLAU, G. (1993), « Testing the relationship of locus of control to different performance dimensions », Journal of Occupational and Organizational Psychology, vol. 66, $\mathrm{n}^{\mathrm{o}} 2$, p. 125-138.

BLICKLE, G., SCHNEIDER, P. B., PERREWÉ, P. L., BLASS, F. R. et FERRIS, G. R. (2008), « The roles of self-disclosure, modesty, and self-monitoring in the mentoring relationship: a longitudinal multi-source investigation », Career Development International, vol. 13, $\mathrm{n}^{\mathrm{o}} 3$, p. 224-240.

BRIGHAM, K. H., DE CASTRO, J. O. et SHEPHERD, D. A. (2007), « A PersonOrganization Fit Model of Owner-Managers' Cognitive Style and Organizational Demands », Entrepreneurship Theory and Practice, vol. 31, n 1, p. 29-51.

BURNS, D. J., REID, J. S., TONCAR, M., FAWCETT, J. et ANDERSON, C. (2006), « Motivations to volunteer: the role of altruism », International Review on Public and Nonprofit Marketing, vol. 3, $\mathrm{n}^{\mathrm{o}}$ 2, p. 79-91. 
BUSSELL, H. et FORBES, D. (2002), « Understanding the volunteer market: The what, where, who and why of volunteering ", International Journal of Nonprofit and Voluntary Sector Marketing, vol. 7, nº 3, p. 244-257.

CLARY, E. G., SNYDER, M., RIDGE, R. D., COPELAND, J., STUKAS, A. A., HAUGEN, J. et MIENE, P. (1998), « Understanding and assessing the motivations of volunteers: a functional approach », Journal of Personality and Social Psychology, vol. 74, $\mathrm{n}^{\mathrm{o}}$ 6, p. 1516.

CLUTTERBUCK, D. (2004), Everyone needs a mentor: Fostering talent at work ( $^{\text {th }}$ éd.), London, U.K., Chartered Institute of Personnel and Development (CIPD), $200 \mathrm{p}$.

CNAAN, R. A. et GOLDBERG-GLEN, R. S. (1991), « Measuring Motivation to Volunteer in Human Services », The Journal of Applied Behavioral Science, vol. $27, n^{\circ} 3$, p. 269-284.

CULL, J. (2006), « Mentoring young entrepreneurs: What leads to success? », International Journal of Evidence Based Coaching and Mentoring, vol. 4, $\mathrm{n}^{\mathrm{o}}$ 2, $\mathrm{p}$. 8-18.

D'ABATE, C. P., EDDY, E. R. et TANNENBAUM, S. I. (2003), « What's in a name? A literature-based approach to understanding mentoring, coaching, and other constructs that describe developmental interactions ", Human Resource Development Review, vol. 2, no 4, p. 360-384.

DESCHAMPS, B., FATIEN, P. et GEINDRE, S. (2010), « Accompagner le repreneur d'entreprise : conduire, escorter mais aussi guider », Gestion 2000, vol. 27, n⿳⺈ 3, p. 77-88.

DINDIA, K. (2002), « Self-disclosure research: Knowledge through meta-analysis », dans M. Allen, R. W. Preiss, B. M. Gayle et N. Burrell (dir.), Interpersonal communication research: Advances through meta-analysis, Mahwah, NJ, Lawrence Erlbaum Associates, p. 169-185.

DWYER, P. C., BONO, J. E., SNYDER, M., NOV, O. et BERSON, Y. (2013), « Sources of volunteer motivation: Transformational leadership and personal motives influence volunteer outcomes », Nonprofit Management and Leadership, vol. 24, $\mathrm{n}^{\mathrm{o}} 2$, p. 181-205.

EGAN, T. M. (2005), « The Impact of Learning Goal Orientation Similarity on Formal Mentoring Relationship Outcomes », Advances in Developing Human Resources, vol. $7, n^{\circ} 4$, p. 489-504.

GARNER, J. T. et GARNER, L. T. (2011), «Volunteering an opinion: Organizational voice and volunteer retention in nonprofit organizations », Nonprofit and Voluntary Sector Quarterly, vol. 40, nº 5, p. 813-828.

GHISLIERI, C., GATTI, P. et QUAGLINO, G. (2009), « Factors affecting willingness to mentor », International Journal for Educational and Vocational Guidance, vol. 9, $\mathrm{n}^{\mathrm{o}} 3$, p. 205-219.

GIBSON, S. K. (2005), « Whose best interests are served? The distinction between mentoring and support ", Advances in Developing Human Resources, vol. 7, $\mathrm{n}^{\mathrm{o}}$ 4, p. 470-488.

HAGGARD, D. L., DOUGHERTY, T. W., TURBAN, D. B. et WILBANKS, J. E. (2011), « Who Is a Mentor? A Review of Evolving Definitions and Implications for Research », Journal of Management, vol. 37, $\mathrm{n}^{\mathrm{o}}$ 1, p. 280-304.

HELLMAN, C. M. (1997), « Job satisfaction and intent to leave », The Journal of Social Psychology, vol. 137, nº 6, p. 677-689. 
HERMAN, K. C. et USITA, P. M. (1994), « Predicting Big Brothers/Big Sisters volunteer attrition with the $16 \mathrm{PF} »$, Child and Youth Care Forum, vol. 23, nº 3, p. 207-211.

HIBBERT, S., PIACENTINI, M. et DAJANI, H. A. (2003), « Understanding volunteer motivation for participation in a community-based food cooperative $»$, International Journal of Nonprofit and Voluntary Sector Marketing, vol. 8, $\mathrm{n}^{\circ} 1$, p. 30-42.

HU, C., WANG, S., YANG, C. C. et WU, T. Y. (2014), « When mentors feel supported: Relationships with mentoring functions and protégés' perceived organizational support », Journal of Organizational Behavior, vol. 35, n ${ }^{\circ}$ 1, p. 22-37.

HUNTER, D. A. (2007), Why Do Mentors Mentor?: A Study of Motivations in Workplace Mentoring. Dissertation, Indiana University of Pennsylvania, Pennsylvania, USA

JANSSEN, S., VAN VUUREN, M. et DE JONG, M. D. (2014), « Motives to mentor: Self-focused, protégé-focused, relationship-focused, organization-focused, and unfocused motives », Journal of Vocational Behavior, vol. 85, $\mathrm{n}^{\circ}$ 3, p. 266-275.

KIM, S. (2007), « Learning goal orientation, formal mentoring, and leadership competence in HRD: A conceptual model », Journal of European Industrial Training, vol. 31, n 3, p. 181-194.

LANGDON, F. J. (2014), « Evidence of mentor learning and development: an analysis of New Zealand mentor/mentee professional conversations », Professional development in education, vol. 40, $\mathrm{n}^{\mathrm{o}} 1, \mathrm{p} .36-55$.

MADIA, B. P. et LUTZ, C. J. (2004), « Perceived Similarity, Expectation-Reality Discrepancies, and Mentors' Expressed Intention to Remain in Big Brothers/Big Sisters Programs "), Journal of Applied Social Psychology, vol. 34, n 3, p. 598623.

MAU, W.-C. J., ELLSWORTH, R. et HAWLEY, D. (2008), « Job satisfaction and career persistence of beginning teachers », International Journal of Educational Management, vol. 22, $\mathrm{n}^{\mathrm{o}} 1$, p. 48-61.

MEISSEN, G. J. et LOUNSBURY, J. W. (1981), « A comparison of expectations of volunteers, children, and parents in a big brother-big sister program », Journal of Community Psychology, vol. 9, no 3, p. 250-256.

MILLER, L. C., BERG, J. H. et ARCHER, R. L. (1983), « Openers: Individuals who elicit intimate self-disclosure », Journal of Personality and Social Psychology, vol. 44, no 6, p. 1234-1244.

MILLETTE, V. et GAGNÉ, M. (2008), « Designing volunteers' tasks to maximize motivation, satisfaction and performance: The impact of job characteristics on volunteer engagement ", Motivation and Emotion, vol. 32, n 1, p. 11-22.

MORROW, K. V. et STYLES, M. B. (1995), Building relationships with youth in program settings: A study of Big Brothers/Big Sisters, Philadelphia, Public/Private Ventures, $126 \mathrm{p}$.

NIEHOFF, B. (2006), « Personality predictors of participation as a mentor », Career Development International, vol. 11, $\mathrm{n}^{\circ}$ 4, p. 321-333.

OKUN, M., BARR, A. et HERZOG, A. (1998), « Motivation to volunteer by older adults: A test of competing measurement models », Psychology and Aging, vol. $13, n^{\circ} 4$, p. 608-621. 
OZGEN, E. et BARON, R. A. (2007), « Social sources of information in opportunity recognition: Effects of mentors, industry networks, and professional forums », Journal of Business Venturing, vol. 22, nº 2, p. 174-192.

PAUL, M. (2004), L'accompagnement : une posture professionnelle spécifique, Paris, France, L'Harmattan, $356 \mathrm{p}$.

PAULHUS, D. (1983), « Sphere-specific measures of perceived control », Journal of Personality and Social Psychology, vol. 44, nº 6, p. 1253-1265.

PAYETTE, A. et CHAMPAGNE, C. (1997), Le groupe de codéveloppement professionnel, Ste-Foy, Québec, Presses de l'Université du Québec.

PULLINS, E. B., FINE, L. M. et WARREN, W. L. (1996), «Identifying peer mentors in the sales force: An exploratory investigation of willingness and ability », Journal of the Academy of Marketing Science, vol. 24, no 2, p. 125-136.

RADU LEFEBVRE, M. et REDIEN-COLLOT, R. (2013), " "How to Do Things with Words": The Discursive Dimension of Experiential Learning in Entrepreneurial Mentoring Dyads », Journal of Small Business Management, vol. 51, n 3, p. 370393.

RAGINS, B. R. et COTTON, J. L. (1993), « Gender and willingness to mentor in organizations », Journal of Management, vol. 19, $\mathrm{n}^{\circ}$ 1, p. 97-111.

RICE, M. B. et BROWN, R. D. (1990), « Developmental Factors Associated with SelfPerceptions of Mentoring Competence and Mentoring Needs », Journal of college student development, vol. 31, $\mathrm{n}^{\circ}$ 4, p. 293-299.

RUDNITSKY, M. (2003), Motivated mentors : an examination of the construct of motivation to mentor, its antecedents and its consequences. Masters thesis, Concordia University, Montréal, Canada

SPECTOR, P. E. (1986), « Perceived control by employees: A meta-analysis of studies concerning autonomy and participation at work », Human Relations, vol. 39, $\mathrm{n}^{\circ}$ 11, p. 1005-1016.

SPECTOR, P. E., COOPER, C. L., SANCHEZ, J. I., O'DRISCOLL, M., SPARKS, K., BERNIN, P., BOSSING, A., DEWE, P., HART, P. et LU, L. (2002), « Locus of control and well-being at work: how generalizable are western findings? », Academy of Management Journal, vol. 45, n 2, p. 453-466.

SPENCER, R. (2007), «"It's Not What I Expected” A Qualitative Study of Youth Mentoring Relationship Failures », Journal of Adolescent Research, vol. 22, n 4, p. 331-354.

SPRECHER, S., TREGER, S., WONDRA, J. D., HILAIRE, N. et WALLPE, K. (2013), « Taking Turns: Reciprocal Self-Disclosure Promotes Liking in Initial Interactions », Journal of Experimental Social Psychology, vol. 49, n 5, p. 860866.

ST-JEAN, E. (2010), «Les fonctions du mentor de l'entrepreneur novice », Revue de l'entrepreneuriat, vol. $9, \mathrm{n}^{\circ}$ 2, p. 28-49.

ST-JEAN, E. (2012), « Mentoring as professional development for novice entrepreneurs: maximizing the learning », International Journal of Training and Development, vol. $16, n^{\circ} 3$, p. 200-216.

ST-JEAN, E. et AUDET, J. (2012), « The role of mentoring in the learning development of the novice entrepreneur », International Entrepreneurship and Management Journal, vol. 8, n⿳⺈ 1, p. 119-140. 
ST-JEAN, E. et AUDET, J. (2013), « The Effect of Mentor Intervention Style in Novice Entrepreneur Mentoring Relationships », Mentoring \& Tutoring: Partnership in Learning, vol. 21, n ${ }^{\circ} 1$, p. 96-119.

ST-JEAN, E. et EL AGY, M. (2012), « La motivation à être bénévole des mentors : un effet auprès des entrepreneurs accompagnés? ». $2^{\text {ème }}$ Rencontre entre les acteurs des réseaux d'accompagnement et chercheurs, Montpellier, France, 19 janvier.

ST-JEAN, E. et MITRANO-MÉDA, S. (Accepté), « Former les mentors pour entrepreneurs pour aller au-delà de la transmission d'expérience », Revue de l'entrepreneuriat.

ST-JEAN, E. et TREMBLAY, M. (2011), «Opportunity Recognition With A Mentor: The Role Of The Entrepreneur's Learning Goal Orientation ». Babson College Entrepreneurship Reseach Conference, Syracuse, N.Y., June $8^{\text {th }}-11^{\text {th }}$.

STATISTIQUE CANADA (2009), Canadiens dévoués, canadiens engagés : points saillants de l'enquête canadienne de 2007 sur le don, le bénévolat et la participation, Statistique Canada, Ottawa, Canada.

STUKAS, A. A. et TANTI, C. (2005), « Recruiting and sustaining volunteer mentors », dans D. L. DuBois et M. J. Karcher (dir.), Handbook of Youth Mentoring, Thousand Oaks, CA, SAGE Publications, p. 235-250.

TABACHNICK, B. G. et FIDELL, L. S. (2007), Using Multivariate Statistics (5 ${ }^{\text {th }}$ éd.), Boston, MA, Allyn \& Bacon, 980 p.

UCBASARAN, D., WESTHEAD, P. et WRIGHT, M. (2009), « The extent and nature of opportunity identification by experienced entrepreneurs », Journal of Business Venturing, vol. 24, $\mathrm{n}^{\mathrm{o}} 2$, p. 99-115.

VAN EMMERIK, H., BAUGH, S. G. et EUWEMA, M. C. (2005), « Who wants to be a mentor? An examination of attitudinal, instrumental, and social motivational components », Career Development International, vol. 10, nº 4, p. 310-324.

VAN GINKEL, G., VERLOOP, N. et DENESSEN, E. (2016), « Why mentor? Linking mentor teachers' motivations to their mentoring conceptions », Teachers and Teaching, vol. 22, $\mathrm{n}^{\mathrm{o}}$ 1, p. 101-116.

WANBERG, C. R., WELSH, E. T. et HEZLETT, S. A. (2003), « Mentoring research: A review and dynamic process model », dans J. J. Martocchio et G. R. Ferris (dir.), Research in personnel and human resources management, Oxford, UK, Elsevier Science Ltd. Vol. 22, p. 39-124.

WANBERG, C. R., WELSH, E. T. et KAMMEYER-MUELLER, J. (2007), « Protégé and mentor self-disclosure: Levels and outcomes within formal mentoring dyads in a corporate context », Journal of Vocational Behavior, vol. 70, n 2, p. 398412.

WANG, S., NOE, R. A., WANG, Z.-M. et GREENBERGER, D. B. (2009), « What affects willingness to mentor in the future? An investigation of attachment styles and mentoring experiences », Journal of Vocational Behavior, vol. 74, $\mathrm{n}^{\mathrm{0}} 3$, p. 245-256.

WYMER, W., RIECKEN, G. et YAVAS, U. (1997), « Determinants of volunteerism: A cross-disciplinary review and research agenda », Journal of Nonprofit \& Public Sector Marketing, vol. 4, $\mathrm{n}^{\mathrm{o}}$ 4, p. 3-26. 